\section{Una red de biobancos para Chile: investigar hoy, para curar mañana}

\author{
JUVENAL A. RÍOS ${ }^{1,6, b}$, ELISA ALCALDE², \\ EUGENIO RAMÍREZ ${ }^{3, a}$, MYRIAM CAMPBELL ${ }^{4}$, \\ TOMAS P. LABBÉ ${ }^{5,9, b}$, SERGIO BECERRA ${ }^{6}$, \\ SYLVIA SANTANDER ${ }^{7}$, MARÍA ELENA CABRERA ${ }^{8}$
}

\section{The implementation of a Biobank network for Chile}

The concept "Biobank" is relatively new in the scientific literature, and is not yet consensually defined, even for the World Health Organization (WHO). However, the use of human samples in biomedical research is a very old activity. The organized development of Biobanks in different places has grown in the last decade. The experience in different countries and continents has been diverse. In this special article we intend to summarize, organize and communicate to the national medical and scientific community, (i) the concept of Biobank, (ii) the international experience and a map of the Research Biobanks working in Chile, (iii) the basic biomedical and essential operational aspects to manage a Biobank for Research and (iv) the impact of a National Network of Biobanks implementation in the Chilean Health System. Ethical and regulatory aspects will not be included, given their intrinsic complexity, which should be discussed elsewhere.

(Rev Med Chile 2019; 147: 901-909)

Key words: Biological Specimen Banks; Biomedical Research; Research.

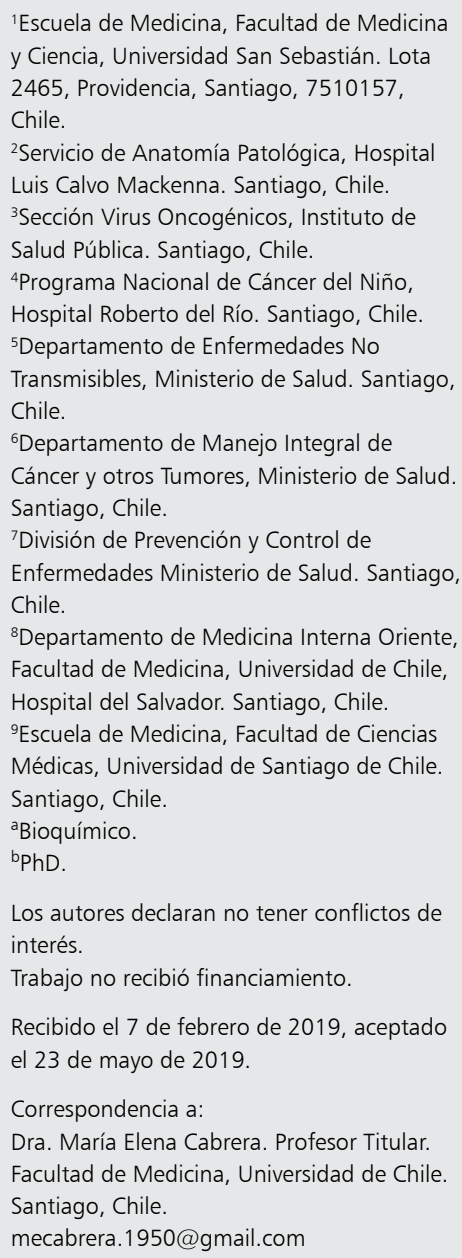

Los autores declaran no tener conflictos de interés.

Trabajo no recibió financiamiento.

Recibido el 7 de febrero de 2019, aceptado el 23 de mayo de 2019.

Correspondencia a:

Dra. María Elena Cabrera. Profesor Titular. Facultad de Medicina, Universidad de Chile. Santiago, Chile.

mecabrera.1950@gmail.com

\section{Consideraciones generales}

\subsection{Orígenes y concepto de biobancos}

$\mathrm{E}$ 1 término biobanco es un concepto relativamente nuevo. La primera vez que se mencionó el término "biobank" en una investigación científica, data del año 1996, en la revista alemana Journal of Molecular Medicine (Berliner Klinische Wochenschrift) ${ }^{1}$. Posteriormente, en 1998, en un artículo danés se mencionó por primera vez el término biobanco en el título de la publicación ${ }^{2}$. Tal ha sido su impacto hasta la fecha, que en el 2009, la revista "Time" eligió a los biobancos como una de las 10 mejores ideas que cambiarán al mundo ${ }^{3}$. En la actualidad, al realizar una búsqueda en la plataforma de PUBMED, es posible encontrar aproximadamente 4.000 artículos con el término "biobank" en el título, observándose, además, un incremento marcado en los últimos cinco años (Figura 1).

Para el desarrollo de este artículo es necesario distinguir los términos biobanco y biorrepositorio. La Organización para la Cooperación y el Desarrollo Económicos (OCDE) define biobancos como "una colección de material biológico asociado a datos e información guardados en un sistema organizado para la población"4. Por otra parte, un biorrepositorio se entiende como la infraestructura dentro de la cual se identifican, recopilan, almacenan y distribuyen muestras biológicas ${ }^{5}$. Al mismo tiempo, la Agencia Internacional para la Investigación en Cáncer (IARC) usa el término 


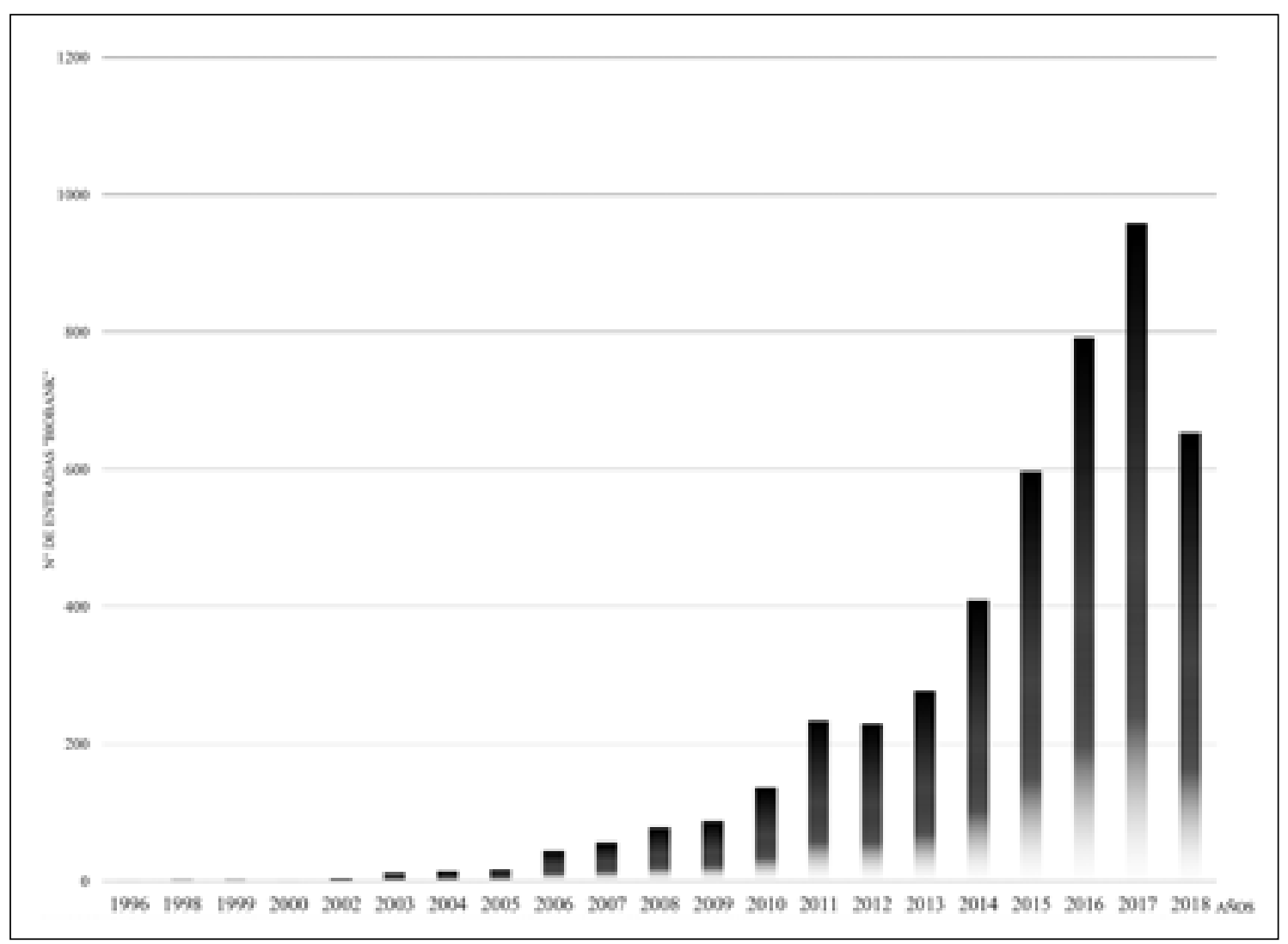

Figura 1. Número de entradas en bases de datos PUBMED al ingresar el término "biobank".

"centro de recursos biológicos" para referirse a las colecciones de muestras de cáncer de origen humano ${ }^{6}$. El Instituto Nacional del Cáncer de Estados Unidos de Norteamérica define a un biorrepositorio como "organización, lugar, pieza o contenedor donde las muestras biológicas son guardadas"'.

En nuestro medio local, y tomando en consideración varios autores ${ }^{8}$, hemos definido biobancos como "organización pública o privada, sin fines de lucro, que almacena y custodia colecciones de material biológico humano asociados a información del estado de salud o los estilos de vida de la persona, para fines de investigación biomédica o epidemiológica y que se organizan como una unidad técnica con criterios de calidad, orden y trazabilidad".

Además, debemos distinguir:

Red de biobancos: Conjunto de biobancos asociados a una entidad central, que actúa como coor- dinador entre ellos. La entidad jurídica pertenece a cada uno de los biobancos de forma individual ${ }^{9}$.

Biobancos en red: Aquellos con una única organización y una actividad descentralizada, situación en que varios biobancos se asocian creando un nodo central que se constituye como figura jurídica única que incluye a todas las demás. Aquí las muestras suelen distribuirse en las diferentes instituciones que componen el biobanco ${ }^{9}$.

\subsection{Breve reseña de la experiencia internacional y nacional}

Estados Unidos de Norteamérica (USA): En 1949 se organizó en el Hospital Naval en Bethesda, Maryland, el primer banco de tejido óseo. Esta unidad se encargó del procesamiento y distribución de injertos óseos durante 50 años, con fines terapéuticos ${ }^{10}$. La Red Cooperativa de Tejidos Humanos de Estados Unidos de América (CHTN, por sus siglas en inglés) se fundó dentro del marco del 
Tabla 1. Mapa de los biobancos de investigación* en Chile

\begin{tabular}{|ll|}
\hline Sistema público & Sistema privado \\
\hline Hospital Sótero del Río & Proyecto “Chile-BILS" - PUC \\
\hline Hospital del Salvador** & Clínica Alemana de Santiago \\
\hline Hospital Luis Calvo Mackenna & Fundación Arturo López Pérez**** \\
\hline Hospital de Molina & \\
\hline Hospital San Borja Arriarán*** & \\
\hline Universidad de la Frontera & \\
\hline Universidad de Chile (BTUCH) & \\
\hline
\end{tabular}

* La gran mayoría son biobancos de tumores. ${ }^{* *}$ Material genético de enfermedades oncohematológicas. ${ }^{* *}$ Sus colecciones están resguardadas en el BTUCH (Proyecto del Grupo Colaborativo Oncológico Cooperativo Chileno de Investigación con el

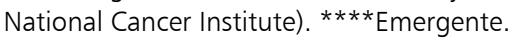

Programa de Diagnóstico de Cáncer del Instituto Nacional de Cáncer en $1987^{11}$. Alrededor de 1.500 publicaciones relacionadas con las alteraciones genéticas en el inicio, la progresión y la metástasis del cáncer y de estudios para mejorar la exactitud diagnóstica y la clasificación de los tumores se han realizado con muestras de esta red ${ }^{12}$.

España: En Madrid, en el año 1998 se creó la Red de Banco de Tumores (RBT) del Centro Nacional de Investigaciones Oncológicas (CNIO) con el apoyo del Instituto de Salud Carlos III y el patrocinio de fundaciones y empresas privadas. Colaboran con la RBT las comunidades de Madrid, Castilla, Galicia, País Vasco, Asturias, Canarias, Andalucía y Murcia, las cuales cuentan con 16 unidades intrahospitalarias ${ }^{13}$.

Brasil: El primer Banco de Tumores fue organizado en 1997 en Sao Paulo, el cual aportó con ejemplares latinoamericanos al proyecto Genoma Humano (1999). Este banco maneja alrededor de 19.000 muestras. En Río de Janeiro funciona el Banco de Tumores y ADN (BNT) del Instituto Nacional del Cáncer (INCA), fundado en 2004, y que cuenta con más de 17.000 muestras de diversos tejidos; el BNT dispone de 20 centros médicos y académicos distribuidos en cinco regiones geográficas del país ${ }^{14}$.

Chile: El primer biobanco se fundó en Temuco el año 2004, en la Facultad de Medicina de la Universidad de la Frontera ${ }^{15}$. En la actualidad se reconoce la existencia de 9 biobancos con fines de investigación. De estos, hay 3 biobancos ubicados fuera de establecimientos públicos: (i) el de la Pontificia Universidad Católica de Chile (PUC) en el Hospital Clínico UC, que está asociado al Proyecto "Chile-BILS"(Chilean Biliary Longitudinal Study), que corresponde a una alianza entre la PUC y la UFRO para estudiar el cáncer de vesícula biliar y enfermedades biliares; (ii) de la Clínica Alemana de Santiago y (iii) otro emergente del Instituto Fundación Oncológica Arturo López Pérez. Por su parte, existen 6 biobancos asociados a instituciones públicas: (iv) del Complejo Asistencial Hospital Sótero del Río; (v) del Hospital del Salvador; (vi) del Hospital Luis Calvo Mackenna; (vii) de la Universidad de la Frontera (UFRO); (viii) de Tejidos y Fluidos de la Universidad de Chile (BTUCH) y (ix) del Hospital de Molina, donde se alojan las muestras de la cohorte de MAUCO del Proyecto Basal FONDAP-ACCDIS ${ }^{17}$. Adicionalmente, estuvo en funcionamiento el biobanco correspondiente al Hospital San Borja Arriarán ${ }^{17}$, pero en la actualidad sus muestras están resguardadas en el BTUCH (Tabla 1).

\subsection{Biobancos en el marco del Plan Nacional de Cáncer 2018-2028}

En el contexto del reciente lanzamiento del Plan Nacional de Cáncer por el Ministerio de Salud de Chile, la existencia de una red nacional de biobancos se ha explicitado ${ }^{18}$. Considerando la necesidad de contar con datos locales de mayor representatividad y de promover la investigación traslacional en cáncer, la existencia de estos centros constituye un sustrato esencial para realizar investigación biomédica de alto impacto, 
teniendo en cuenta que, en los últimos años, la participación de los médicos en actividades de generación de nuevo conocimiento parece estar en retroceso ${ }^{19}$.

\section{Aspectos biomédicos y de gestión, fundamentales para un biobanco con fines de investigación}

\subsection{Los biobancos como un servicio público}

En contraposición a las colecciones de material biológico personal o también llamados por algunos autores como "biorrepositorios", los biobancos deben ser un servicio público, sin fines de lucro, con consentimiento amplio, control social $\mathrm{y}$ altos estándares profesionales ${ }^{20}$.

\subsection{Tipos y diversidad de biobancos}

Por un lado, existen biobancos para estudiar biomarcadores de susceptibilidad y de exposición, en los cuales se busca tener datos genético-epidemiológicos de poblaciones y su interacción con el ambiente. Por otro lado, existen los biobancos para buscar biomarcadores de enfermedad y esencialmente de tumores, aspecto por lo cual son más conocidos ${ }^{21}$. Asimismo, cabe mencionar que también existen diversos subtipos de biobancos, entre los que se incluyen de cerebros, VIH, múltiples agentes infecciosos, tejidos sanos, organoides, exososomas, incluso de algunos destinados solo a enfermedades raras ${ }^{22}$.

\subsection{Estructura hospitalaria}

La organización intrahospitalaria es fundamental, dado que es el lugar físico y administrativo donde a menudo se instalan los biobancos. Cabe destacar que los biobancos no constituyen una "curiosidad" de interés para patólogos, hematólogos o los investigadores básicos. Estas instituciones están al servicio de la comunidad científica y hospitalaria, y sometidos a un control de gestión riguroso que garantice la calidad de su funcionamiento. También es importante destacar el rol del profesional coordinador (enfermera $\mathrm{u}$ otro profesional de la salud), ente fundamental en el registro de muestras biológicas humanas ${ }^{23,24}$.

\subsection{Procedimientos normalizados, buenas prácticas y recurso humano entrenado}

Los procedimientos de un biobanco deben estar escritos, guiados por la experiencia in- ternacional y prudentemente adaptados a la realidad local. Además, deben ser consensuados por equipos multidisciplinarios y revisados periódicamente $e^{6,25,26}$.

\subsection{Tamaño de muestra versus calidad}

Mientras que la cantidad de muestras es un factor discutible y teóricamente limitante en la mantención de los biobancos, la calidad de ellos es definitivamente el factor clave de las conclusiones de los estudios y, por tanto, los posteriores beneficios e impactos en salud 6 .

\subsection{Los biobancos no son almacenes}

El principio fundamental de la existencia de los biobancos es para ceder o compartir muestras y no son para almacenar. El biobanco de excelencia es probablemente aquel que mantiene un volumen estable de muestras, pero al mismo tiempo cede muchas para proyectos de investigación relevantes en salud y con real impacto sanitario ${ }^{25}$.

\subsection{Información clínica asociada}

Del mismo modo que la información clínica de los pacientes es esencial para la interpretación de cualquier examen en su contexto apropiado, el biobanco necesita recopilar información asociada a la muestra para poder proveer de insumos completos a los investigadores. Esta información no corresponde solo a datos clínicos, sino también epidemiológicos e histopatológicos ${ }^{27}$. Esta entidad binominal -muestra/historial clínico- impone desafíos éticos relevantes; en la elaboración del consentimiento informado para efectos de entregar la donación y su posterior utilización, entorno a la privacidad y confidencialidad, uso secundario de muestras y datos para estudios futuros, retorno de resultados, intercambio de datos, manejo de datos genéticos de una población, distribución de beneficios, recontacto de donantes y envío de muestras al extranjero, entre otras ${ }^{28}$.

\subsection{Trabajo colaborativo}

La colaboración local y extranjera resultan fundamentales para crecer en número de datos y así obtener mejores conclusiones. Esta cooperación también ofrece una oportunidad de consensuar protocolos e intercambiar formación de recurso humano que permita garantizar la calidad del funcionamiento de un biobanco ${ }^{29,30}$. 


\subsection{Sostenibilidad económica}

Contar con muestras de alto valor potencial y calidad implica una gran inversión en recurso humano e infraestructura. Sin embargo, aun a nivel mundial no hay consenso respecto a la forma de financiar los biobancos ${ }^{31}$. Un estudio del Servicio Nacional de Salud del Reino Unido, el año 2009, demostró que $80 \%$ de los laboratorios $(\mathrm{n}=700)$ tenían serias dificultades para obtener muestras estandarizadas $^{31}$. La actividad de los biobancos es heterogénea, ya que puede ser realizada por universidades, sistema público de salud o entidades privadas, lo que dificulta encontrar un modelo común de sostenibilidad económica ${ }^{32}$. Los costos varían dependiendo del tipo de muestra, como se almacenan y conservan, si se obtuvo de un procedimiento diagnóstico o terapéutico, a través de un protocolo de rutina o una intervención específica, el transporte y distribución, si es un tejido fresco o no, el registro y las bases de datos asociadas, entre otros factores ${ }^{32}$.

En general, hay consenso a nivel mundial que no está permitido vender muestras de material biológico humano con fines lucrativos. Sin embargo, sí se acepta recuperar los costos de recolectar, procesar, almacenar y distribuir el material biológico ${ }^{31}$.

\subsection{El donante es todo}

El paciente es el principio y el final en los biobancos. Al comienzo es el donante y finalmente debería ser quien se beneficie directa o indirectamente de la información derivada de las investigaciones $^{33}$.

\section{Potencial impacto de una red nacional de biobancos en planificación sanitaria}

Los biobancos desarrollan una función importante en el desarrollo de la investigación biomédica, en la comprensión los procesos de enfermedad, en el desarrollo de la medicina personalizada. Con el advenimiento de la medicina preventiva de precisión, influyen en la planificación sanitaria por medio de la utilización de datos “ómicos", entendiéndose estos como todo aquello que comprenda información de tipo genómica (estudio integral del funcionamiento, el contenido, la evolución y el origen de los genomas), transcriptómica (estudio de las moléculas ARN celular), proteómica (estudio de la estructura y función de las proteínas), metabolómica (estudio del metabolismo, es decir, los procesos de conversión de energía de los alimentos en energía mecánica o calor) y epigenómica (estudio de las modificaciones química y topológicas, por efectos internos y ambientales, que experimenta la cromatina) $)^{34,35}$.

Uno de los campos mayormente beneficiados por el conocimiento generado desde los biobancos es la oncología ${ }^{36}$, en particular la oncología de precisión, en la cual también aplican las $4 \mathrm{P}$ de Leroy Hood, esto es "personalizada, preventiva, predictiva y participativa" ${ }^{37}$. En este sentido enumeramos algunos hallazgos que han tenido impacto en la historia natural de la enfermedad:

1. Prevención y tamizaje: búsqueda de nuevos biomarcadores. En el caso específico del cáncer de próstata esto es de especial relevancia, dada la cuestionada utilidad del antígeno prostático específico (APE) como predictor de este tipo de tumor ${ }^{38}$.

2. Diagnóstico: búsqueda de nuevos biomarcadores diagnóstico para cáncer de colon, páncreas y tiroides ${ }^{39-41}$.

3. Pronóstico: generación de perfiles genéticos para mutación del gen KIT, para predecir sensibilidad a drogas como imatinib ${ }^{42}$.

4. Tratamiento: contribución en predicción de reacciones adversas y posología correcta en pacientes sometidos a quimioterapia ${ }^{43}$.

Cabe señalar que la mayoría de los biobancos en Europa y USA son financiados de forma mixta: estatal y por organizaciones que los cobijan, tales como universidades u hospitales. Así, el conocimiento obtenido de las investigación se transforman en un bien público, lo cual debería redundar finalmente en el beneficio de la salud de las personas ${ }^{44}$. Sin embargo, la investigación tiene un largo camino desde un hallazgo fundamental hasta generar un impacto visible en la salud de personas y poblaciones. Se estima que este proceso tarda alrededor de 10 a 15 años. Esta latencia es un importante obstáculo que desincentiva a las compañías farmacéuticas a invertir en investigación y desarrollo ${ }^{45}$.

\section{Conclusiones y Desafíos}

Podemos resumir los siguientes aspectos en que los biobancos impactan el desarrollo y mejo- 
ramiento de la salud humana: (i) son esenciales para la identificación de nuevos biomarcadores y terapias específicas, (ii) influyen en el progreso de la investigación genómica, lo que ha llevado a distinguir enfermedades multifactoriales de forma más precisa y (iii) al relacionar grupos cooperativos de investigación internacional se incrementa el número de casos estudiados, permitiendo obtener mejores conclusiones en enfermedades crónicas prevalentes como el cáncer y las enfermedades cardiovasculares, pero también constituyen una fuente invaluable de información en el caso de las enfermedades raras ${ }^{46}$.

Por otra parte, disponer de muestras biológicas de calidad es crítico para el descubrimiento, validación y uso del conocimiento generado por los biobancos. Esta situación representa un enorme desafío desde el punto de vista de los costos y financiamiento de estas instituciones.

Para mantener un adecuado equilibrio entre sostenibilidad y la actividad "no lucrativa" intrínseca de los mismos, planteamos las siguientes medidas de funcionamiento:

a) Que la adquisición de la muestra sea financiada por proyectos, asegurando que el objetivo del biobanco sea dirigido por necesidades de investigación en salud.

b) La actividad del biobanco y el equipamiento sea financiado con fondos públicos distribuido a las instituciones que los albergan (universidades u hospitales).

c) Que el acceso a las muestras sea pagado por el usuario con una tarifa, que implica una recuperación del costo.

Chile tiene una oportunidad única de desarrollo de los biobancos, dada su heterogeneidad geográfica, climática, étnica y socio-demográfica, generando diferencias en los perfiles epidemiológicos de al menos 5 macrozonas (extremo norte, norte, centro, sur y extremo sur). Esta situación está presente tanto en población sana, en riesgo, como en la enferma propiamente tal. Tener una imagen detallada y a gran escala de la biología de nuestra población, permitirá generar datos precisos para instaurar adecuadas políticas de prevención, diagnóstico precoz, tratamiento y rehabilitación. La implementación de políticas sanitarias, incluyendo la información "ómica", permitirá a Chile dar un salto cuántico en la manera de implementar la salud pública, impulsando a posicionar nuevamente nuestros indicadores sanitarios al nivel de los países desarrollados.

Agradecimientos: Agradecemos al renovado equipo del Departamento de Manejo Integral de Cáncer y Otros Tumores del Ministerio de Salud de Chile; Maritza, María José, Fanny, Gina, Priscilla y Dunja. Por su labor incansable al servicio público y por otorgarnos el tiempo para escribir literatura científica, en paralelo a las contingencias que día a día conlleva el trabajo en el Ministerio de Salud. Asimismo, la autora Dra. Cabrera agradece especialmente a EU María Lea Derio, fundadora de la Unidad de Cáncer en el Ministerio de Salud y quien les encomendó y motivó a desarrollar esta nueva línea de trabajo.

\section{Referencias}

1. Loft S, Poulsen HE. Cancer risk and oxidative DNA damage in man. J Mol Med (Berl) [Internet]. 1996 Jun [citado el 22 de diciembre de 2018]; 74 (6): 297-312. Available from: http://www.ncbi.nlm.nih.gov/pubmed/8862511.

2. Nørgaard-Pedersen B. [The PKU registry and biobank at the National Serum Institute. Rules and applications]. Ugeskr Laeger [Internet]. 1998 Apr 6 [citado el 22 de diciembre de 2018]; 160 (15): 2266-7. Available from: http://www.ncbi.nlm.nih.gov/pubmed/9599526.

3. TIME Magazine Cover: 10 Ideas Changing the World Right Now - Mar. 23, 2009 - Environment - Economy Africa - Religion [Internet]. [citado el 29 de diciembre de 2018]. Available from: http://content.time.com/time/ covers/0,16641,20090323,00.html.

4. Patil S, Majumdar B, Awan KH, Sarode GS, Sarode SC, Gadbail AR, et al. Cancer oriented biobanks: A comprehensive review. Oncol Rev [Internet]. 2018 Jan 30 [citado el 29 de diciembre de 2018]; 12 (1): 357 . Available from: http://www.ncbi.nlm.nih.gov/pubmed/30057691

5. Mora E, A. Robb J, Stefanoff G, Mellado R, Coppola D, Muñoz-Antonia T, et al. Proceedings of the $1^{\text {st }}$ Puerto Rico Biobanking Workshop. Rev Recent Clin Trials [Internet]. 2015 Mar 6 [citado el 29 de diciembre de 2018]; 9 (4): 233-44. Available from: http://www.eurekaselect.com/openurl/content.php?genre=article\&iss$\mathrm{n}=1574-8871$ \&volume $=9$ \&issue $=4$ \&spage $=233$.

6. Mendy M, Caboux E, Lawlor RT, Wright J, Wild CP. Common minimum technical standards and protocols for biobanks dedicated to cancer research IARC Technical Publication No. 44. https://publications.iarc.fr/ 
Book-And-Report-Series/larc-Technical-Publications/ Common-Minimum-Technical-Standards-And-Protocols-For-Biobanks-Dedicated-To--Cancer-Research-2017 [citado el 29 de diciembre de 2018]. 101 p. Available from: http://publications.iarc.fr/551.

7. NCI Best Practices for Biospecimen Resources. National Cancer Institute 2016. Available from: https://biospecimens.cancer.gov/bestpractices/2016-NCIBestPractices. pdf.

8. Marodin G, França P, Rocha JCC DA, Campos AH. Biobanking for health research in Brazil: present challenges and future directions. Rev Panam Salud Pública [Internet]. 2012 [citado el 29 de diciembre de 2018]; 31 (6): 523-8. Available from: http:// www.scielosp.org/scielo.php?script=sci_arttext\&pi$\mathrm{d}=$ S1020-49892012000600012\&lng=en\&nrm=iso\&tln$\mathrm{g}=\mathrm{en}$.

9. Decreto Real $1716 / 201$, de 18 de noviembre por el que se establecen los requisitos básicos de autorización y funcionamiento de los biobancos con fines de investigación biomédica y del tratamiento de las muestras biológicas de origen humano, y se regula el funcionamiento $y$ organización del Registro Nacional de Biobancos para investigación biomédica. BOE $n^{\circ} 290$ de 2 de diciembre de 2011.

10. Strong DM. The US Navy Tissue Bank: 50 Years on the Cutting Edge. Cell Tissue Bank [Internet]. 2000 [citado el 29 de diciembre de 2018]; 1 (1): 9-16. Available from: http://www.ncbi.nlm.nih.gov/pubmed/15256965.

11. CHTN - Cooperative Human Tissue Network [Internet]. [citado el 29 de diciembre de 2018]. Available from: https://www.chtn.org/.

12. RFA-CA-18-025: Collaborative Human Tissue Network (CHTN) (UM1 Clinical Trials Not Allowed) [Internet]. [citado el 29 de diciembre de 2018]. Available from: https://grants.nih.gov/grants/guide/rfa-files/rfa-ca-18-025. html.

13. Centro Nacional de Investigaciones Oncológicas - CNIO [Internet]. [citado el 29 de diciembre de 2018]. Available from: https://www.cnio.es/.

14. Camargo AC. Cancer Center [Internet]. [citado el 29 de diciembre de 2018]. Available from: https://www. accamargo.org.br/.

15. Roa EI, Artigas ACG. Banco de tumores en Chile, su aporte a la investigación: resultados de un proyecto piloto. Rev Med Chile [Internet]. 2008 [citado el 29 de diciembre de 2018]; 136 (6): 733-40. Available from: http://www.scielo.cl/scielo.php?script=sci_arttext\&pi$\mathrm{d}=$ S0034-98872008000600007\&lng=en\&nrm=iso\&tln$\mathrm{g}=\mathrm{en}$.

16. Ferreccio C, Roa JC, Bambs C, Vives A, Corvalán AH,
Cortés S, et al. Study protocol for the Maule Cohort (MAUCO) of chronic diseases, Chile 2014-2024. BMC Public Health [Internet]. 2016 [citado el 29 de diciembre de 2018]; 16: 122. Available from: http://www.ncbi. nlm.nih.gov/pubmed/26847446.

17. Se inaugura primer Banco de Tumores de la red pública de salud de Chile | Instituto de Salud Pública de Chile [Internet]. [citado el 29 de diciembre de 2018]. Available from: http://www.ispch.cl/noticia/19208.

18. Ministerio de Salud G de C. Plan Nacional de Cáncer 2018-2028 [Internet]. 2018 [citado el 4 de enero de 2019]. Available from: https://cdn.digital.gob.cl/filer public/d3/0a/d30a1f5e-53d9-4a31-a4fe-e90d8d9a2348/ documento_plan_nacional_de_cancer.pdf.

19. Labbe T, Barake F, Alamos MF, Molina C, Ríos J. Realidad de la Investigación en Salud en Chile: participación de los Médicos en el Fondo Nacional de Investigación (FONIS) en la última década. ARS MEDICA Rev Ciencias Médicas [Internet]. 2017 [citado el 4 de enero de 2019];42(2):76-80. Available from: http://www.arsmedica.cl/index.php/MED/article/view/933.

20. Liaño F, Torres A. Biobancos: una nueva herramienta para la investigación clínica. Nefrología [Internet]. 2009 Jun 1 [citado el 29 de diciembre de 2018]; 29 (3): 193 5. Available from: http://www.revistanefrologia.com/ es-biobancos-una-nueva-herramienta-investigacion-articulo-X0211699509004575.

21. Harris JR, Burton P, Knoppers BM, Lindpaintner K, Bledsoe M, Brookes AJ, et al. Toward a roadmap in global biobanking for health. Eur J Hum Genet [Internet]. 2012 [citado el 29 de diciembre de 2018]; 20 (11): 110511. Available from: http://www.nature.com/articles/ ejhg201296.

22. Henderson GE, Cadigan RJ, Edwards TP, Conlon I, Nelson AG, Evans JP, et al. Characterizing biobank organizations in the U.S.: results from a national survey. Genome Med [Internet]. 2013 [citado el 29 de diciembre de 2018]; 5 (1): 3. Available from: http://genomemedicine.biomedcentral.com/articles/10.1186/gm407.

23. Riegman PHJ, de Jong B, Daidone MG, Söderström T, Thompson J, Hall JA, et al. Optimizing sharing of hospital biobank samples. Sci Transl Med [Internet]. 2015 [citado el 29 de diciembre de 2018]; 7 (297): 297fs31. Available from: http://www.ncbi.nlm.nih.gov/ pubmed/26203078.

24. Seiler CY, Eschbacher J, Bowser R, LaBaer J. Sustainability in a Hospital-Based Biobank and University-Based DNA Biorepository: Strategic Roadmaps. Biopreserv Biobank [Internet]. 2015 [citado el 29 de diciembre de 2018]; 13 (6): 401-9. Available from: http://www.ncbi. nlm.nih.gov/pubmed/26697909. 
25. OECD Guidelines on Human Biobanks and Genetic Research Databases Organisation for Economic Co-Operation and Development [Internet]. 2009 [citado el 29 de diciembre de 2018]. Available from: www.oecd.org/ publishing/corrigenda.

26. Certified Repository Technician Training Program ISBER [Internet]. [citado el 29 de diciembre de 2018]. Available from: https:/www.isber.org/page/CRT/Certified-Repository-Technician-Training-Program.htm.

27. Cui W, Zheng P, Yang J, Zhao R, Gao J, Yu G. Integrating Clinical and Biological Information in a Shanghai Biobank: An Introduction to the Sample Repository and Information Sharing Platform Project. [citado el 29 de diciembre de 2018]; Available from: http://www. stem-art.com/Library/Biobanking/Integrating Clinical and Biological Information in a Shanghai Biobank - An Introduction to the Sample Repository and Information Sharing Platform Project.pdf.

28. Comisión Ministerial de Ética de la Investigación en Salud. BIOBANCOS: Recomendaciones sobre aspectos fundamentales a considerar para el Consentimiento Informado. Ministerio de Salud de Chile. 2017.

29. Zawati MH, Knoppers B, Thorogood A. Population biobanking and international collaboration. Pathobiology [Internet]. 2014 [citado el 29 de diciembre de 2018]; 81 (5-6): 276-85. Available from: http://www.ncbi.nlm.nih. gov/pubmed/25792216.

30. Chen H. Governing International Biobank Collaboration: A Case Study of China Kadoorie Biobank. Sci Technol Soc [Internet]. 2013 [citado el 29 de diciembre de 2018]; 18 (3): 321-38. Available from: http://journals. sagepub.com/doi/10.1177/0971721813498497.

31. Gee S, Oliver R, Corfield J, Georghiou L, Yuille M. Biobank Finances: A Socio-Economic Analysis and Review. Biopreserv Biobank [Internet]. 2015 [citado el 23 de diciembre de 2018]; 13 (6): 435-51. Available from: http://www.liebertpub.com/doi/10.1089/bio.2015.0030.

32. Henderson M, Simeon-Dubach D, Albert M. Finding the Path to Biobank Sustainability Through Sound Business Planning. Biopreserv Biobank [Internet]. 2015 [citado el 23 de diciembre de 2018]; 13 (6): 385-6. Available from: http://www.liebertpub.com/doi/10.1089/ bio.2015.29039.mh.

33. Zawati MH, Lang M. Biobank donors and the concept of benefit: time for reciprocity. J Law Biosci [Internet]. 2017 [citado el 29 de diciembre de 2018]; 4 (2): 371-6. Available from: http://academic.oup.com/jlb/article/4/2/371/3897150/Biobank-donors-and-the-conceptof-benefit-time-for.

34. Kinkorová J. Biobanks in the era of personalized medicine: objectives, challenges, and innovation. EPMA Jour- nal 2016. Available from: https://www.ncbi.nlm.nih.gov/ pmc/articles/PMC4762166/pdf/13167_2016_Article_53. pdf.

35. Caenazzo L, Tozzo P. Biobanks and Public Health: A New Challenge for Public Engagement and Trust. J Biomed Clin Res [Internet]. 2016 [citado el 29 de diciembre de 2018]; 9 (1): 17-20. Available from: https://content. sciendo.com/view/journals/jbcr/9/1/article-p17.xml.

36. Paskal W, Paskal AM, Dębski T, Gryziak M, Jaworowski J. Aspects of Modern Biobank Activity - Comprehensive Review. Pathol Oncol Res [Internet]. 2018 [citado el 29 de diciembre de 2018]; 24 (4): 771-85. Available from: http://link.springer.com/10.1007/s12253-018-0418-4.

37. Hood L, Friend SH. Predictive, personalized, preventive, participatory (P4) cancer medicine. Nat Rev Clin Oncol [Internet]. 2011 [citado el 29 de diciembre de 2018]; 8 (3): 184-7. Available from: http://www.nature.com/ articles/nrclinonc.2010.227.

38. Shui IM, Lindström S, Kibel AS, Berndt SI, Campa D, Gerke T, et al. Prostate cancer (PCa) risk variants and risk of fatal PCa in the National Cancer Institute Breast and Prostate Cancer Cohort Consortium. Eur Urol [Internet]. 2014 [citado el 29 de diciembre de 2018]; 65 (6): 1069-75. Available from: http://www.ncbi.nlm.nih. gov/pubmed/24411283.

39. Nagar S, Ahmed S, Peeples C, Urban N, Boura J, Thibodeau $B$, et al. Evaluation of genetic biomarkers for distinguishing benign from malignant thyroid neoplasms. Am J Surg [Internet]. 2014 [citado el 29 de diciembre de 2018]; 207 (4): 596-601. Available from: http://www. ncbi.nlm.nih.gov/pubmed/24713092.

40. Letellier E, Schmitz M, Baig K, Beaume N, Schwartz C, Frasquilho S, et al. Identification of SOCS2 and SOCS6 as biomarkers in human colorectal cancer. Br J Cancer [Internet]. 2014 [citado el 29 de diciembre de 2018]; 111. Available from: http://www.ncbi.nlm.nih.gov/geo/.

41. Jenkinson C, Elliott V, Menon U, Apostolidou S, Fourkala OE, Gentry-Maharaj A, et al. Evaluation in pre-diagnosis samples discounts ICAM-1 and TIMP-1 as biomarkers for earlier diagnosis of pancreatic cancer. J Proteomics [Internet]. 2015 [citado el 29 de diciembre de 2018]; 113: 400-2. Available from: https://www.sciencedirect.com/science/article/pii/S1874391914004643?via\%3Dihub.

42. Allegra M, Giacchero D, Segalen C, Dumaz N, Butori C, Hofman V, et al. A new KIT mutation (N505I) in acral melanoma confers constitutive signaling, favors tumorigenic properties, and is sensitive to imatinib. J Invest Dermatol [Internet]. 2014 [citado el 29 de diciembre de 2018]; 134 (5): 1473-6. Available from: http://www.ncbi. nlm.nih.gov/pubmed/24317392. 
43. Bielinski SJ, Olson JE, Pathak J, Weinshilboum RM, Wang L, Lyke KJ, et al. Preemptive Genotyping for Personalized Medicine: Design of the Right Drug, Right Dose, Right Time-Using Genomic Data to Individualize Treatment Protocol. Mayo Clin Proc [Internet]. 2014 [citado el 29 de diciembre de 2018]; 89 (1): 25-33. Available from: http://emerge.mc.vanderbilt. edu.

44. De Clercq E, Kaye J, Wolf SM, Koenig BA, Elger BS. Returning Results in Biobank Research: Global Trends and Solutions. Genet Test Mol Biomarkers [Internet]. 2017 [citado el 29 de diciembre de 2018]; 21 (3): 128-
31. Available from: http://www.ncbi.nlm.nih.gov/pubmed/28146646.

45. Ouellette S, Tassé AM. P(3)G-10 years of toolbuilding: From the population biobank to the clinic. Appl Transl genomics [Internet]. 2014 [citado el 29 de diciembre de 2018]; 3 (2): 36-40. Available from: http://www.ncbi. nlm.nih.gov/pubmed/27275412.

46. Botti G, Franco R, Cantile M, Ciliberto G, Ascierto PA. Tumor biobanks in translational medicine. J Transl Med [Internet]. 2012 [citado el 23 de diciembre de 2018]; 10: 1. Available from: http://www.translational-medicine. com/content/10/1/204. 